\title{
Peculiarities the navigation satellites signals scattering in the layered structure of the tree stand
}

\author{
Vladimir Podoprigor $a^{1,3,}{ }^{,}$, Anatoly Sorokin ${ }^{2}$, Dmitriy Kharlamov ${ }^{2}$ \\ ${ }^{1}$ L.V.Kirensky Institute of Physics SB RAS, Krasnoyarsk, Russian Federation \\ ${ }^{2}$ Federal Research Center KSC SB RAS, Krasnoyarsk, Russian Federation \\ ${ }^{3}$ Siberian Federal University, Krasnoyarsk, Krasnoyarsk, Russian Federation
}

\begin{abstract}
A scattering and attenuation processes of navigation satellite signals in the forest interaction with tree trank and crown layers a tree stand are presented. The influence of the boundaries soil - tree stand crown - air on the characteristics of radio signals scattering after interaction with forest is considered. Structural and electro physical features of tree stand elements in the processes of interaction with signals of the $L 1$ range and experimental data on signal transformations the forest are analyzed.
\end{abstract}

\section{Introduction}

In recent years, for the purpose of remote sensing of the earth's covers, radio wave measurements have been successfully used, effectively using the capabilities of the global navigation satellite system. To extract information about micro- and macro parameters of tree stands from satellite signals processed after passing through the tree stand. It is necessary to use a model of a electromagnetic field interaction with tree elements. It is known that at present there is no universal mechanism that takes into account: the multiscale of the forest environment, different radio frequency ranges, the configuration of the experiment, the spatial distribution of trees, the orientation of branches, wood moisture, dielectric properties of wood, bark, leaves and needles. This explains the large number of works that, to one degree or another, explain the features of the passage of a radio signal in a forest, but carried out at various objects in different conditions [1-2]. However, the interaction of radio emission with a tree stand is considered mainly for radar measurements at very low and very high frequencies and without taking into account the influence on the parameters of orientations of tree elements, anisotropy and interfaces of different media.

The peculiarity of the satellite radiation we use is that the wavelength in the L1-range of radio frequencies $(1.5-1.6 \mathrm{~Hz}, \lambda \approx 19 \mathrm{~cm})$ is much larger than the transverse dimensions of the needles and leaves, but is comparable to the diameters of the trunks and thick branches of a tree. In this frequency range, the peculiarities of the internal structure of trees are revealed, which makes it possible to recognize their species and types of tree crowns

\footnotetext{
* Corresponding author: podoprigora46@,gmail.com
} 
using remote sensing. The maximum effective scattering area coefficient for conifers is also observed at these frequencies.

The purpose of this work is to study the parameters of the scattering of signals from L1band navigation satellites from the selected layers of tree trunks and crowns, taking into account the influence on these parameters of the orientation of tree elements and the boundary "soil - layers of trunks and crowns.

The scattering and attenuation characteristics were investigated for forward scattering of radio signals from GLONASS and GPS navigation satellites at a frequency of 1.5-1.6 GHz. The description of the measuring equipment and the scheme of the experimental study are given earlier [3].

\section{Model of interaction the navigation satellites signals with the tree stand layers.}

The tree elements were represented as discrete scatterers, which, in contrast to the continuous medium model, makes it possible to detect spatial inhomogeneities (density fluctuations) in the tree stand. A model description of the interaction of NS signals with a forest assumes the selection of areas with a certain ratio of the sizes of tree elements to the wavelength of radio waves. This allows the correct use of the corresponding approximation. Mechanisms of scattering and attenuation of radio waves in the upper and lower parts of the tree are significantly different. It is natural to select as the above areas the layer of trunks and the layer of crowns, consisting of branches and needles or leaves.

Each layer of the tree stand differs from the other in density, shape, size of tree elements and their orientation in space. The trunks layer is considered as infinite dielectric cylinders, large branches - as cylinders of finite length, needles - highly elongated ellipsoids. To describe small scatterers, the generalized Rayleigh - Hans - Debye (RGD) approximation for ellipsoids was used, which requires the relation $k_{0} d(n-1)<<1$, where $k_{0}$ $=2 \pi / \lambda$ is the wave number in free space, $d$ is the smallest size of the ellipsoid, $n$ is the refractive index. The scattering medium is more transparent for radio waves at least in the direction for one of the ellipsoid axes.

Scattering on trunks and thick branches of trees corresponds to the approximation of geometric optics for infinite and finite cylinders [4] with the latter's size restrictions: $h>>r$, $k_{0} h>>1$, where $h$ and $r$ are the height and radius of the cylinder. It was assumed that the internal field of a finite scatterer can be considered as a field inside an infinite cylinder. When solving the diffraction problem on a tree trunk, this approximation is fulfilled already at $h / r>5$ [4].

An important role in the process of radio wave propagation in the forest is played by the orientation of the scatterers relative to the incident and scattered rays. It can be taken into account in the framework of the tiered approach [5]. The position of the crown branches in space is described by the tensor of orientational ordering, and the components of the scattering cross section tensor are proportional to the square of the anisotropy and the components of this tensor. Here, the tiers play the role of crown sublayers, in each of which there is a uniform distribution of thick branches along the azimuthal angle. The angle of their inclination to the vertical changes with a sinusoidal distribution function from $30^{\circ}$ to $120^{\circ}$ - from the upper to the lower tiers. A separate layer or tier of the crown consists of groups of scatterers, each of which is characterized by a certain size and orientation of thin branches with its own distribution function. 


\section{Boundaries of layers in a tree stand}

At sufficiently high frequencies (above $1 \mathrm{GHz}$ ), the description model of the scattering and attenuation processes of radio waves in a forest can include irregularities of different scales of interfaces, such as "soil - tree trunks", "crown - air", "tree bark - air". These irregularities can affect the scattering characteristics when considering the expressions responsible for the interaction of waves with the indicated boundaries. The shape of the reflecting surface of these boundaries is always irregular, and the height of the points of the surface $h(x, y)$ is described by a random function of coordinates (statistically rough surface). It is usually considered that the distribution of heights $h$ obeys the normal law with the mathematical expectation $\langle h(x, y)\rangle=0$ and the variance $\delta_{h}{ }^{2}$. The spatial correlation function $L_{h}=\left\langle h\left(x_{1}, y_{1}\right) \times h\left(x_{2}, y_{2}\right)\right\rangle$ is assumed to be isotropic; a Gaussian curve is often used for it.

A surface is considered to be fine-rough if the height $h(x, y) / \lambda$ and its partial derivatives with respect to the $x$ and $y$ coordinates are much less than one. In some cases, such surfaces include the boundary "soil - layer of trunks". For the scattering matrix, the well-known Kirhoff model (with the constraint $h / \lambda<0,3$ ) can be applied.

Large-scale irregularities (for example, the boundary "air - crowns" for the $L 1$-frequency) are characterized by the radii of curvature $R_{x, y}>>\lambda$, while no limitation is imposed on their heights. For large irregularities $\delta_{h} / \lambda=h / \lambda>>1$, variance $h^{2} \leq \lambda^{2}$ or $h^{2} \geq \lambda^{2}$. With these assumptions the scattering characteristics can be calculated.

The study shows that if, when determining the components of the scattering matrix, the interactions of waves with elements of crowns, trunks and soil surface are taken into account. Soil irregularities can significantly change the angular dependence of these components. So, modeling of the dependence of the specific effective scattering area (SESA) on the angle of incidence, taking into account the interaction of waves with the soil and the layers of the tree stand, shows the same values of $\square_{0}$ for a smooth and rough surface in the range of incidence angles $\square=5^{\square}-7 \square$. In the region $\square=30^{\circ}-40^{\circ}$, the difference in the SESA values reaches almost $9 \mathrm{~dB}$, the contributions to scattering from interaction with the soil are higher for an uneven surface than for a flat one, and strongly depend on the size of roughness and the polarization of the incident radiation.

\section{Radioscopy the volume of larch forest by signals a navigation satellites}

Registration of a scattered and attenuation signal by a larch stand (the Pogorelsky Bor station, Institute of Forest, KSC SB RAS) was carried out by a receiving antenna with a vertically up top oriented pattern. The antenna was located above the soil at a height of 4.2 $\mathrm{m}$. The amplitude of the signal-to-noise ratio of right circular polarization and the angular coordinates of the satellites with a frequency of $1 \mathrm{~Hz}$ were recorded. The duration of the session was 23.6 hours. Inhomogeneities in the distribution of trees elements in the radio scanning zone of the tree stand transformed the parameters signals of navigation satellites.

The heterogeneity of the tree elements distribution in the area of radio scanning of the tree stand has the following characteristics: stand density -0.114 trees $/ \mathrm{m} 2$, average values of height and diameter are 26 and 0,19 meters respectively. The boundaries of the crown layer are located at heights from $12-13$ to 25-26 meters and have vertical gradients biomass. The trunks layer occupies space from the soil to the gradient border with the height of the trank trees, at which the trunk diameter is comparable to the diameters of the main branches and is located at a height of 20-23 meters. The space at heights from 12 to 22 meters occupy parts layers crowns and tree trunks. The scanning signals of navigation 
satellites sequentially propogate through the both layers. The ratio of contributions to the change in signals parameters depends on the height of the receiving antennas and the elevation angle

The separation of the contributions to the field of scattered radiation by the crown layer and trunks is in principle possible with the use of coordinated electrophysical models of the layers and the technique of measuring the characteristics of the signals transmitted by the tree stand.

The graphs of space-time dependences of the signals characteristics of the GLONASS 9 navigation satellite after passing throuh thr forest shown in Fig. 1 and Fig. 2.

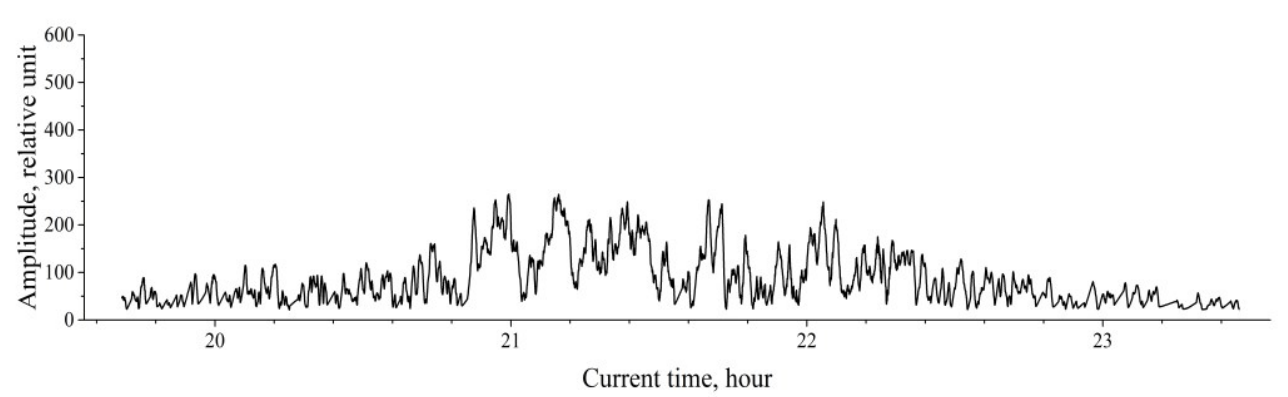

Fig 1. The space-time dependences amplitude of the signals after tree stand, session № 1

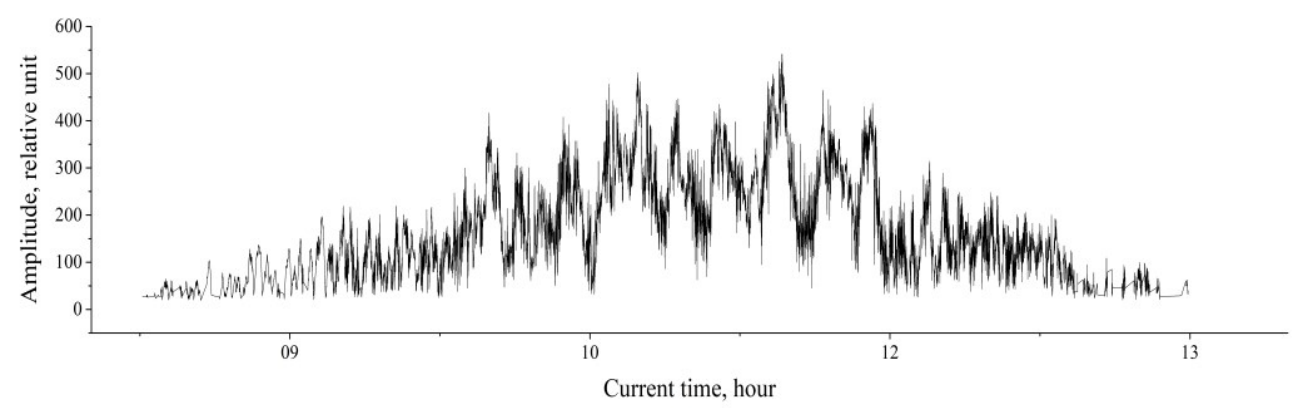

Fig 2. The space-time dependences amplitude of the signals after tree stand, session № 2

The tree stand was scanned in the sectors of azimuthal angles: session $1\left(103^{\circ}-19^{\circ}\right)$ and session $2\left(303^{\circ}-190^{\circ}\right)$ with maximum elevation angles $31^{\circ}$ and $75^{\circ}$, respectively. Option 1 demonstrates greater attenuation when driving through the forest compared to option 2 . The Amplitude variations of the scanning signal are random and determined by the inhomogeneities of the tree stand.

The coherence of the probe radiation and the chaotically inhomogeneous distribution of the effective dielectric constant of the crown and trunk layers ensure the formation of a speckle structure in the radiation field scattered by the medium. A large amount of experimental data with a coordinate reference $[5,6,10)]$ makes it possible to assess the state of local forest volumes.

In the process of scanning the forest stand with navigation satellite signals, it is effective to use different radio illumination options. These features are realized by moving the signal source and continuously registering the satellite's angular coordinates. The polar diagram of the angular coordinates of GLONASS 9 relative to the receiving antenna during radio illumination is shown in Fig. 3. The presented curves in figures 1 and 2combination with the coordinate data of the antenna location, the position of the radiation source and the 
General taximetric characteristics of the tree stand allow you to determine the location of the scanning volumes and the linear attenuation coefficients in them.

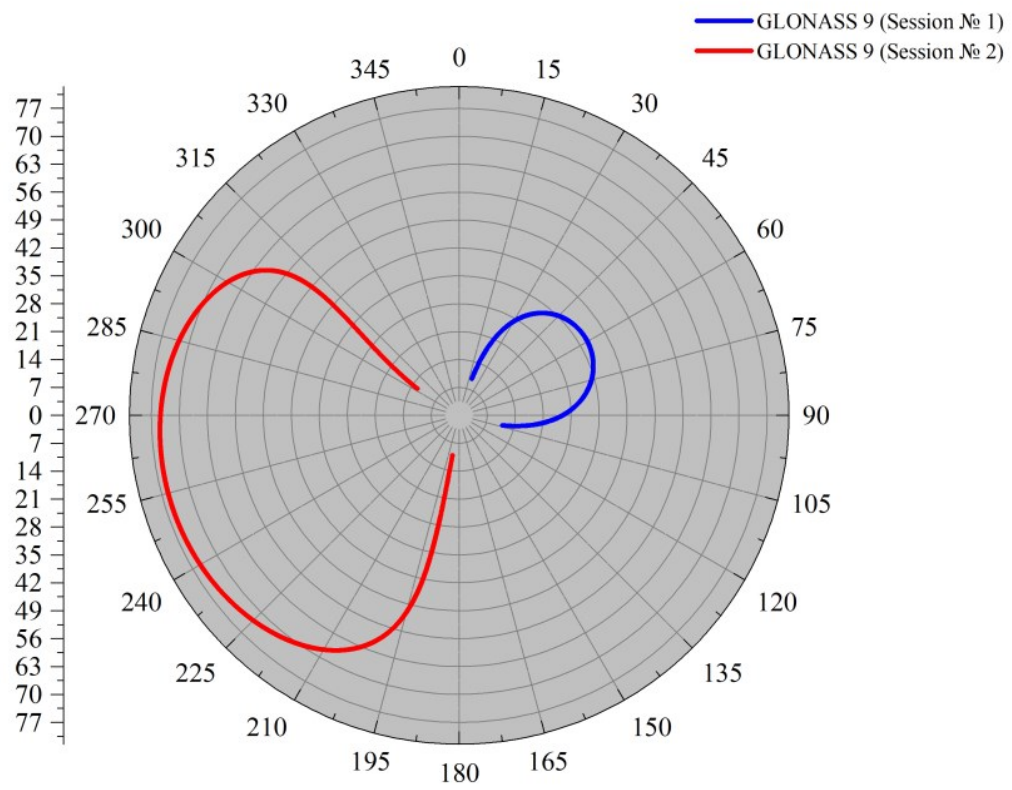

Fig. 3. The angular coordinates of GLONASS 9 in the process of radio transmission of the forest.

The complexity of modeling the propagation of radio waves in the forest is known $[1,2$, $7,8]$. The prospects of using the signals of navigation satellites of the L1 range as a universal instrumental measuring resource and adaptive modeling of the forest stand, taking into account its layered structure, are real. An important factor in the implementation of the method for restoring the electro physical characteristics of the forest is to take into account the polystructure of the tree stand, [9], the anisotropy of the trunk layer [10] and the fractal structure of the crowns [11].

\section{Conclusions}

Different-scale groups of scatterers in the crowns of trees with respect to the wavelength of a radio wave in the L 1 range $(\sim 19 \mathrm{~cm})$ were identified: small branches, needles, leaves; large branches taking into account their spatial orientation.

The randomly inhomogeneous distribution of the effective dielectric constant of the crown and trunk layers in combination with the coherence of signals from navigation satellites provides the formation of the speckle structure of the radiation scattered by the forest.

An example of the space-time dependence of the field value at the point of location of the receiving antenna after the passage of the signal from the navigation satellite of the larch stand is presented. 
A variant of restoring the electrophysical characteristics of the tree stand using signals from navigation satellites and modeling the process of propagation of radio waves, taking into account the polystructurality of the stand, anisotropy of the stem layer] and the fractal structure of crowns is real.

\section{References}

1. V.I.. Popov, Propagation of radio waves in forests.-M. Hotline-Teletom. (2015).

2. A. Chukhlantsev. A.M Shutko., S.P Golovanov. Radio engineering and electronics., V 48, No. 11, p.1285-1311 (2003)

3. V. Podoprigora, A. Sorokin. E3S Web Conf.,Volume 75, 01007, Regional Problems of Earth Remote Sensing (RPERS 2018) https://doi.org/10.1051/e3sconf/20197501007. (2019)

4. S. Seker, A. Schreider. IEEE Trasn.Antennas Propagat. 1988, V 36, p.303-307.

5. A. Sorokin, A Ostylovsky, A. Borisov, V. Ivanov, D. Makarov, D Kharlamov. E3S Web of Conferences 149, 00 https://doi.org/10.1051/e3sconf/20201490 RPERS 2019 2062006 (2020)

6. V. Podoprigora, A. Sorokin, D. Makarov, D. Kharlamov. E3S Web of Conferences 149, 00 https://doi.org/10.1051/e3sconf /20201490 RPERS 20192052005 (2020)

7. S. Novik, A. Zavyalov, E. Telpukhovsky, Izvestiya Vuzov. Physics. V.49, № 7, P.5767. (2006)

8. A. Sorokin, V. Podoprigora, S. Fomin, I Savin. Izvestiya vuzov. Physics.. V. 60. № 12/2. pp. 19-22, (2017)

9. G. Karev, Structural models of forest ecosystems. Siberian Ecological Journal. 4 pp. 381-396. (1999)A.

10. Sorokin, V. Podoprigora,, D. S. Makarov, D.V. Kharlamov, V.V. Baltais. Proceedings of higher educational institutions. Physics. V. 63, No. 2. P. 50-54 (2020)

11. V. Yakubov, N. Lisenko. Izvestiya vuzov. Physics.. V. 55. № 12/2. pp. 19-22, pp 259261. (2012) 